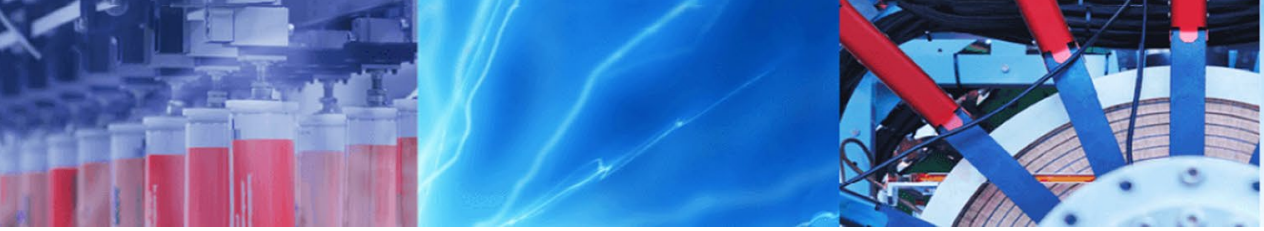

Review Paper

\title{
Dual connectivity for LTE-NR cellular networks in evolved packet system and critical review on challenges in SgNB release
}

\author{
Martin Kollar ${ }^{1}$ (D) \\ (c) Springer Nature Switzerland AG 2019
}

\begin{abstract}
The inter-generations dual connectivity (DC) between the $4 G$ and $5 G$, named as LTE-NR, has got a lot of attention in 3GPP Release 15 because such integration may help to guarantee $5 \mathrm{G}$ dedicated demands concerning high throughput, reliability, retainability and availability. This paper gives an overview on the DC technology coupling LTE and new radio in evolved packet system and focuses on some problems concerning the SgNB initiated SgNB release due to user inactivity reasons.
\end{abstract}

Keywords Dual connectivity $\cdot$ New radio $\cdot$ MeNB $\cdot$ SgNB $\cdot$ EN-DC

\section{Introduction}

Over last decade the number of devices and diversity of services carried by the mobile network requesting both high-quality and high throughput services drastically increased which resulted in a significant growth in mobile traffic. This resulted into improvements of $4 \mathrm{G}$ technology but also developing some $5 \mathrm{G}$ revolutionary technical innovations like network slicing allowing multiple logi$\mathrm{cal} / \mathrm{virtual}$ networks to be created on the top of a common shared physical infrastructure to reach the increased capacity and needs of each group of services [1, 2].

The high user throughput requirements can be achieved by means of Heterogeneous 'Networks (HetNets) $[2,3]$. The HetNets architecture is composed of macro and micro cells. One macro cell involves more than one small cell and have therefore bigger coverage compared to small cell. The small cells in this architecture are acting as hotspot areas increasing capacity and help to dispose of some user plane data from the macro cell $[2,4,5]$. The disadvantage of such a deployment is that the needed number of small cells is higher which significantly increases also the number of mobility procedures like cell reselections and handovers which may result in mobility-related issues.

In order to fix the mobility-related issues concerning the HetNets, the DC technology has been standardized in 3GPP Release 15 [2, 6]. A User Equipment (UE) in DC configuration can be connected at the same time to two Node Bs: a Master eNB (MeNB) and a Secondary gNB (SgNB), which act on different carriers and are connected by $\mathrm{X} 2$ interface. According to 3GPP Release 12 similar constellation can be achieved for intra LTE case when a User Equipment (UE) which is configured with DC can be connected at the same time to two Evolved Node Bs (eNBs): a Master eNB (MeNB) and a Secondary eNB (SeNB).

In Fig. 1, an example of a DC scenario coupling LTE and NR is illustrated. It consists of a MeNB interconnected with two SgNBs via X2 links and three UEs. UE-1 and UE-2 are connected to MeNB and SgNB-2, respectively, while the UE-3 is in DC with the SgNB-1 and MeNB. Therefore, the throughput of UE-3 may be increased because radio resources from SgNB-1 and MeNB are utilized by this UE $[2,7,8]$.

Communication at DC scenario coupling LTE and NR, however, introduces new challenges for the whole

Martin Kollar, Martin.Kollar@nokia.com | ${ }^{1}$ MBB FDDLTE SDA O\&M 2 PL, Nokia Solutions and Networks, Sp. z o.o, Strzegomska 36, 53611 Wrocław, Poland. 


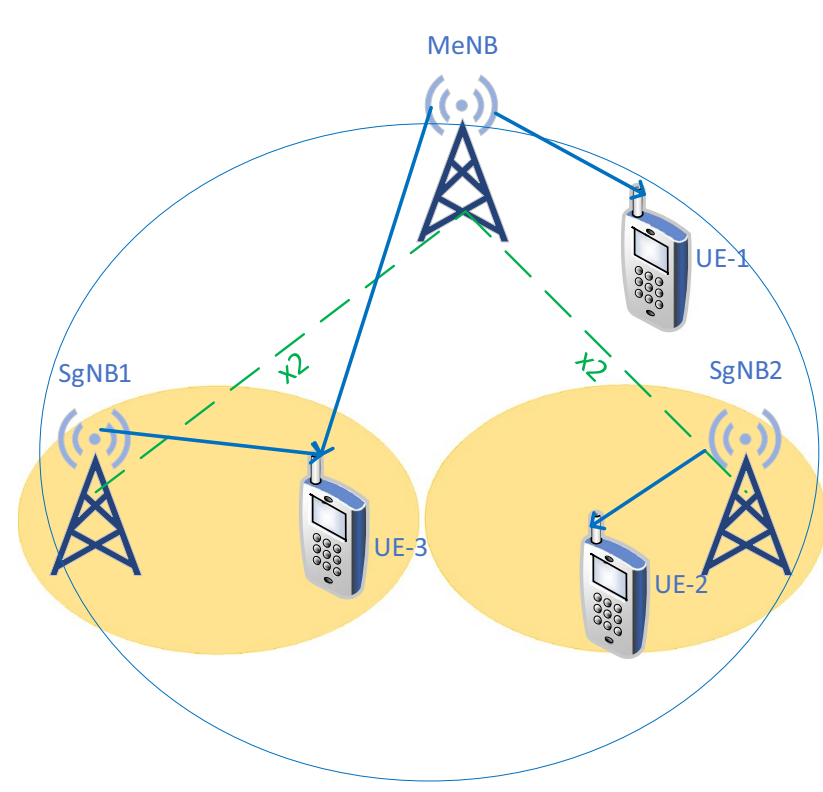

Fig. 1 Example of DC scenario consisting of one MeNB and two SgNBs

protocol stack, which may affect the end-to-end performance and the quality of experience perceived by the users. Besides the main issues at NR mmWave frequencies related to the high propagation loss and the sensitivity [9] also bearer management requires some optimization. In DC scenario coupling LTE and NR a concept of split bearers that use the radio protocols located in both MeNB and SgNB was agreed but also a concept of non-split bearers using either the radio protocols located in MeNB or SgNB is still possible $[7,8]$. For instance, let us consider it is SgNB which is monitoring user activity of split bearers and in case there is no user inactivity on any bearer established for the UE in SgNB side, the SgNB may initiate the SgNB release procedure which is always accepted by MeNB. However, the UE may have some other non-split bearers in MeNB that could be reconfigured to split bearer type and thus benefit from LTE-NR DC. Hence, to follow with SgNB release and immediately with new SgNB addition to the same SgNB is not optimal solution as may cause not needed interruption in providing DC services. Therefore, reliable networks will need optimized bearer management operations.

This paper gives an overview on the DC technology coupling LTE and NR in Evolved Packet System, and discusses some challenges concerning the SgNB initiated SgNB release due to user inactivity reasons in such scenario.

The paper has the following structure. Section 2 provides some basic information concerning the system architecture of the DC technology between LTE and NR in Evolved Packet System. In Sect. 3, the basic procedures for the new DC in accordance with 3GPP TS 37.340 [10] and

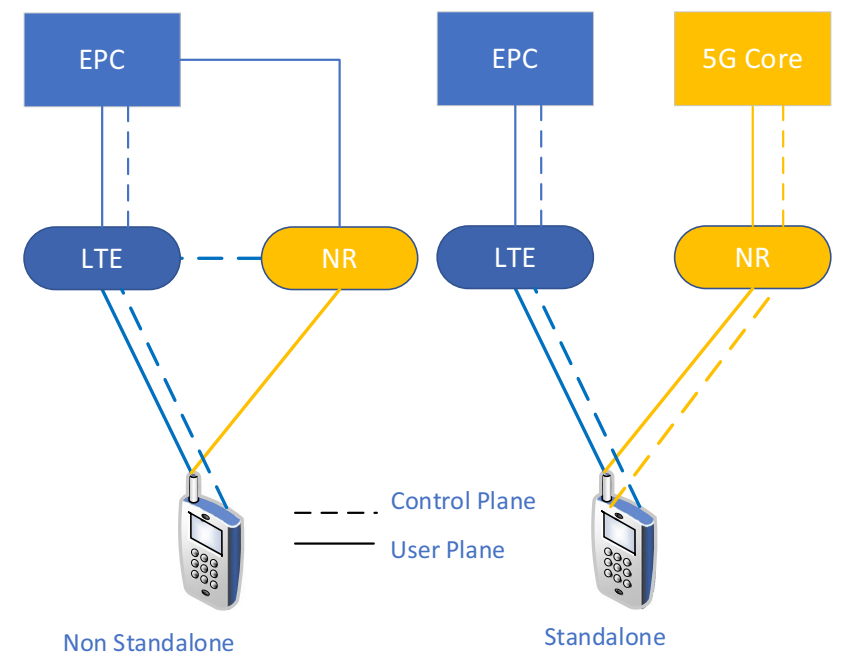

Fig. 2 Principles of NSA and SA operational modes

3GPP TS 36.423 [11] are presented. Challenges concerning the SgNB initiated SgNB release due to user inactivity reasons in the LTE-NR DC scenario are presented in Sect. 4. In Sect. 5 experimental results are presented. Finally, in Sect. 6 , the conclusions and recommendations are drawn.

\section{System architectures for dual connectivity}

The first deployment of $5 \mathrm{G}$ networks is expected under Non-Standalone (NSA) operation, which according to the 3GPP Release 15 specification means that the $5 \mathrm{G}$ networks will reuse existing $4 \mathrm{G}$ infrastructure. This means that $5 \mathrm{G}$-enabled devices will connect to NR cells for user plane data but will still use $4 \mathrm{G}$ for control plane related procedures. Principles of NSA and Standalone (SA) operational modes are shown in Fig. 2.

This paper focuses on DC technology between LTE and NR in Evolved Packet System (EPC) in NSA (LTE eNB anchor, NR gNB anchor) where the architectural Option $3 / 3 A / 3 X$, also called as EN-DC, is possible as indicated in Fig. 3 [12].

This option is suitable at the beginning of $5 \mathrm{G}$ NR introduction for the operators preferring legacy eNB and EPC. As shown in Fig. 3, the MeNB is connected to the EPC, and the NR User Plane (UP) connection to the SgNB goes through the LTE eNB (Option 3) or directly (Option 3A and $3 \mathrm{X})$. X2 interface needs to support Control Plane (CP) and $5 \mathrm{G}$ NR user traffic transmission between MeNB and SgNB for option 3, CP transmission between MeNB and SgNB for option $3 A$ and $3 X$, and split $5 G$ NR user traffic for option $3 X$ [12]. Remaining architectural options possible for NSA and SA operational modes as described in [12].

As depicted in Fig. 3 there are Master Cell Group (MCG) bearer, Secondary Cell Group (SCG) bearer, MCG Split and 


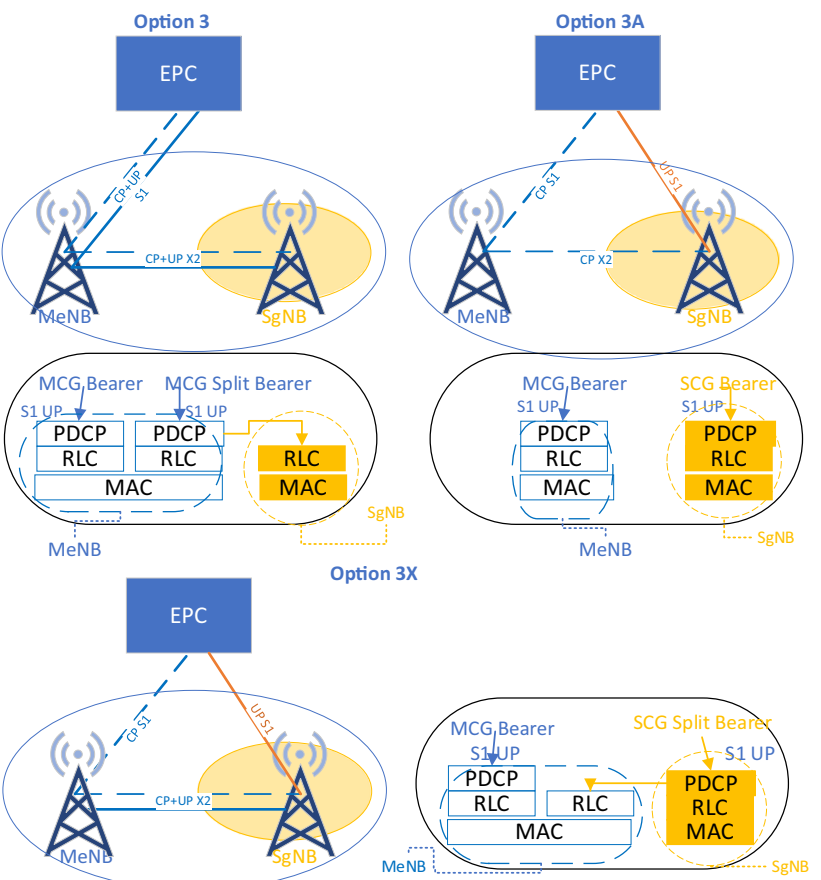

Fig. 3 Architectural options $3 / 3 \mathrm{~A} / 3 \mathrm{X}$

SCG split bearer that can be defined for DC. The MCG/SCG bearer use the resources and protocols located in MeNB/ SgNB only. MCG/SCG Split bearer uses the radio protocols located in both MeNB and SgNB.

\section{Basic procedures for dual connectivity according to 3GPP Release 15}

The DC related procedures were standardized in [10]. The part of the communication between the MeNB and SgNB via X2 interface for DC related procedures was standardized in [11] in Release 15. Because this paper aims to focus on some challenges in SgNB initiated SgNB Release in the sense that such a release is inefficient as immediately followed with SgNB addition meaning the two procedures in addition can be repeated successively certain number of times which may lead to ping pong effect (as it is studied in the Sect. 4) in the following we focus just on SgNB addition and SgNB initiated SgNB release related procedures.

In Fig. 4, an overall signaling procedure to establish UE context at the SgNB is shown. The procedure can be divided into the 9 steps. In the first step the MeNB sends SgNB Addition Request to SgNB. If SgNB accepted the request, it sends SgNB Addition Request Acknowlege back to MeNB in the second step. In the third step the MeNB generates RRC Connection Reconfiguation message with all the necessary information and send it to UE. After UE received RRCConnectionReconfiguration, it checks if all

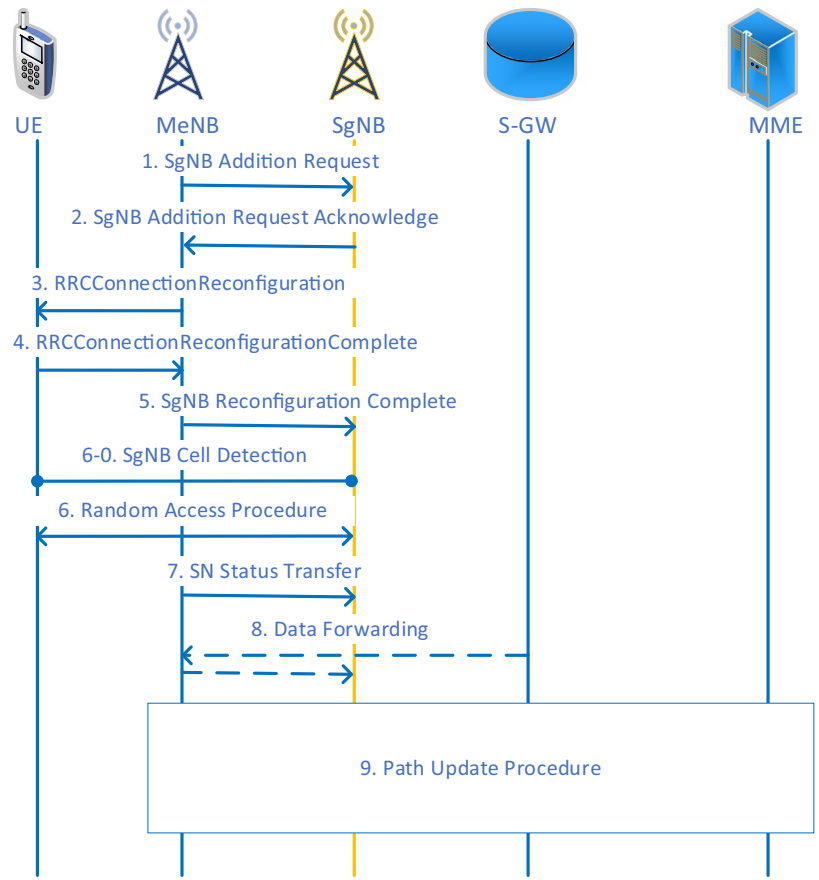

Fig. 4 Overall signaling to establish UE context at the SgNB in accordance with 3GPP TS 37.340

the configurations in the message is doable in UE side and if so, it sends RRCConnectionReconfigurationComplete message back to MeNB as part of the step 4. In the step 5 the MeNB then informs the SgNB about successful completion of the reconfiguration procedure by UE. Based on the information contained in RRCConnectionConfiguration within RRC Connection Reconfiguration message, the UE then detects the NR cell as part of the step 6-0. And after successful detection the UE then performs RACH procedure to NR Cell of the SgNB. In the step 7 MeNB delivers to SgNB PDCP Sequence Number (SN) receiver and transmitter status and starts in DL direction with data forwarding to SgNB as part of the step 8. In the step 9 the Path Update Procedure is executed which encapsulates the IP address of the SgNB delivery to Evolved Packet Core (EPC). After successful completion of the procedure the user data are sent directly from EPC to SgNB and then to UE.

In Fig. 5, an overall signaling procedure to release UE context from the SgNB is shown. The release procedure as depicted in the Fig. 5 is initiated be SgNB. Note it can also be initiated be MeNB. The procedure can be divided into the 8 steps. In the first step the SgNB sends SgNB Release Required to MeNB. According to 3GPPTS 36.423 the MeNB has always to accept it (MeNB cannot refuse this request) and confirms it via sending SgNB Release Confirm to SgNB in the second step. In the third step the MeNB generates RRC Connection Reconfiguation message carrying all the necessary information and send it to UE. After UE received 


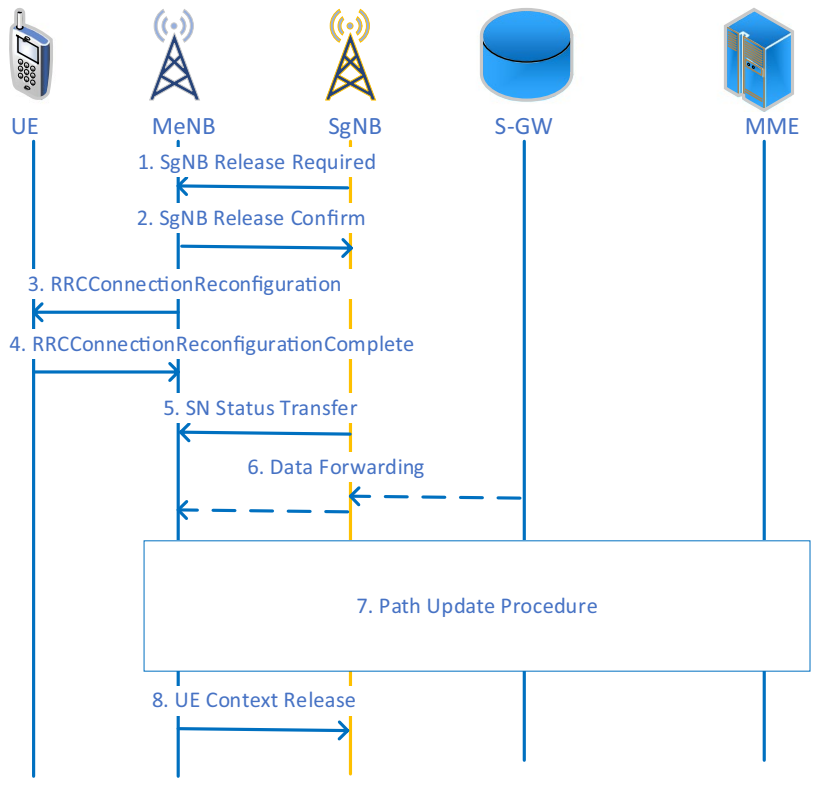

Fig. 5 Overall signaling procedure to release UE context from the SgNB in accordance with 3GPP TS 37.340

RRCConnectionReconfiguration, it sends RRCConnectionReconfigurationComplete message back to MeNB as part of the step 4. In the step 5 SgNB delivers to MeNB PDCP Sequence Number (SN) receiver and transmitter status and starts in DL direction with data forwarding to MeNB as part of the step 6. In the step 7 the Path Update Procedure is executed which encapsulates the IP address of the MeNB delivery to EPC. After successful completion of the procedure the user data are sent directly from EPC to MeNB and then to UE. The procedure is finalized by sending UE Context Release message from MeNB to SgNB in the step 8. The need for all the message exchanges between MeNB and SgNB depends on the reason of the SgNB release. In case it is due to User inactivity the steps 5, 6 and 7 may be skipped and MeNB follows with sending UE Context Release (step 8) to SgNB after step 4.

From performance and capacity management perspective it may be interesting to know the time interval needed to execute the procedures from the Figs. 4 and 5. There are some studies like in [13] pointing out that concerning the Fig. 4 if a $5 G$ NR is integrated with a LTE eNB via DC, and the control protocol (i.e. RRC) is located in the LTE eNB, the control plane latency will be the same as in the LTE case and propose to make this time interval comparable with reference call setup procedure for Control Plane latency in E-UTRAN which shall be less than $35 \mathrm{~ms}$. This value, however, does not include $\mathrm{X} 2$ backhaul delay which is expected to be up to $20 \mathrm{~ms}$ per one message exchange between MeNB and SgNB. In this context, the total time interval to execute the procedure sin Figs. 4 and 5 involving also $\mathrm{X} 2$ backhaul delay can be expected on the range of hundreds of $\mathrm{ms}$. In the remaining part of the paper it is called as interruption time.

\section{Challenges in SgNB initiated SgNB release}

As described in the Sect. 2 the MCG/SCG bearer use the resources and protocols located in MeNB/SgNB only. Configuring these bearers depends on data flow control between the MeNB and SgNB. An efficient flow control mechanism is therefore needed in the MeNB to use the SgNB resources optimally not only for the option 3 but also for reconfiguring MCG bearers to SCG and SCG split bearers because LTE eNB is acting in DC as a master. Regarding this flow control mechanism, some studies exist for intra LTE DC in [14-18] that could be used as basis for LTE-NR DC. In [19] an overview of the work done in this area for LTE-NR DC can be found.

This paper does not aim to contribute to data flow control schemes only refer to them concerning some challenges related to SgNB Release procedure in accordance with Fig. 5. For the Option $3 A / 3 X$ it is SgNB which is monitoring user activity of SCG and SCG split bearers and in case there is no user inactivity on any bearer established for the UE in SgNB side, the SgNB may initiate the SgNB release procedure. According to 3GPPTS 36.423 the MeNB has no power to refuse or reject this requirement from $\mathrm{SgNB}$ and therefore has always to follow with procedures in Fig. 5 [11]. The scenario when the UE has some other MCG bearers that would benefit from LTE-NR DC and the UE is within the coverage of the same SgNB seems to be problematic. This is because to follow with $\mathrm{SgNB}$ release and immediately with SgNB addition to the same SgNB according to Figs. 4 and 5, respectively, is pointless. The total time interval to execute the procedures in Figs. 4 and 5 which is expected not to exceed $50 \mathrm{~ms}$ also represents interruption time interval in providing LTE-NR DC which may negatively impact QoS of the impacted UE. Despite the interruption time interval per one UE does not need to be that critical, however, repeating it couple of times for the same UE and globally for huge number of other UEs it may cause some capacity issues on X2 interface because the number of messages to be exchanged between MeNB and $\mathrm{SgNB}$ is quite a number.

3GPP made a great effort to clarify also this issue and minimize its impact. However, the user story of the 3GPP preferred way to solve it does not need to be clear for all the vendors. The current status, some debates within 3GPP concerning refusal of the SgNB initiated SgNB release from MeNB side are still observed.

3GPP TS 36.423 in the chapter 8.7.16 introduced SgNB Activity Notification as a message sent from SgNB to MeNB 
(see Fig. 6) to notify MeNB on user data traffic activity of all established E-RABs and UE as the whole [11]. It is achieved by means of User plane traffic activity report IE in the SgNB Activity Notification message which is generated for each established E-RAB and the whole UE context.

User plane traffic activity report is shown in Table 1 and in case all the E-RABs established are inactive, the "IE type and reference" is set to inactive for the whole UE context and sent within the SgNB Activity Notification from SgNB to MeNB.

The 3GPP TS 37.340 does not specify any details how MeNB shall react on reception of UE context inactivity info from SgNB [10]. There is just mentioned in the chapter 10.12.1 in [10] that "The MeNB decides to keep SgNB resources" in this case. Anyhow, reception of such a message by the MeNB shall be then understood as prewarning in the sense that SgNB initiated SgNB release due to user inactivity may follow shortly in case the MeNB does not react properly to prevent this release procedure. Under proper reaction a quick procedure initiation from MeNB side to establish new SCG or SCG split bearer (s) if applicable for the UE and the UE is within the coverage of the same SgNB is meant. Establishment of new bearers may be accomplished by means of MeNB initiated SgNB Modification according to 3GPP TS 36.423 [9] and 37.340 [10] as depicted in Fig. 7. In the first step the MeNB sends to MeNB the SgNB Modification Request message containing all needed info for MCG bearer ( $\mathrm{s}$ ) to be reconfigured to SCG ones. SgNB confirms it via sending SgNB Modification Request Acknowledge message to SgNB in the second step. Remaining procedures are like the ones that apply for establishment UE context at the SgNB according to Fig. 4.

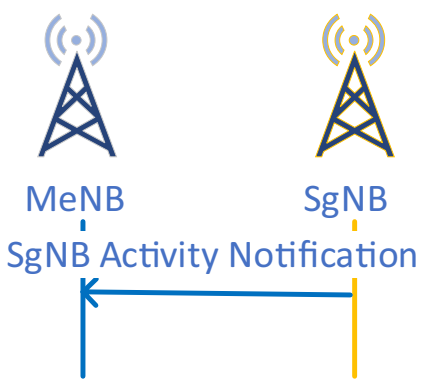

Fig. 6 Activity notification procedure according to 3GPP TS 36.423

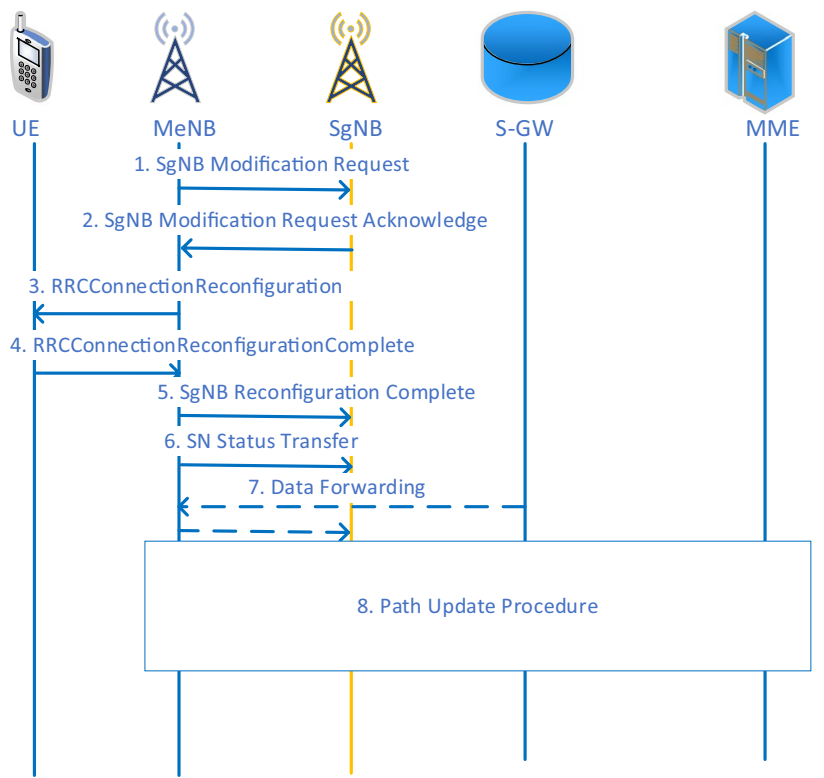

Fig. 7 Overall signaling procedure related to MeNB initiated UE context modification at the SgNB in accordance with 3GPP TS 37.340

Following the steps mentioned in the previous paragraph it shall significantly reduce the interruption time, guarantee seamless continuation of the LTE-NR DC and reduce number of X2AP message exchanges between the MeNB and SgNB.

\section{Experimental results}

To prove the hypothesis from Sect. 4 related to reduction of the interruption time some laboratory experiments and observations were done in a network with deployed DC between LTE on the $2.1 \mathrm{GHz}$ frequency band and NR in the 3.5 GHz frequency band concerning option 3X. As UE a 5G user equipment simulator provided by PRISMA Telecom Testing was used. Primary focus was on absolute difference in interruption time (InterruptionDelta) between the option based on SgNB release followed with SgNB addition (marked as alternative 1 in the next part of the paper) and SgNB Activity Notification followed with SgNB Modification procedure (marked as alternative 2 in the next part

Table 1 User plane traffic activity report in accordance with chapter 9.2.130 in the 3GPP TS 36.423

\begin{tabular}{|c|c|c|c|c|}
\hline IE/group name & Presence & Range & IE type and reference & Semantics description \\
\hline User plane traffic activity report & M & & ENUMERATED (inactive, re-activated, ...) & $\begin{array}{l}\text { "Re-activated" shall be only set after } \\
\text { "inactive" has been reported for the } \\
\text { concerned reporting object }\end{array}$ \\
\hline
\end{tabular}


of the paper) in accordance with details mentioned in the Sect. 4.

The interruption time for the alternative 1 was obtained as sum of three sub intervals according the following equation:

$$
\begin{aligned}
\text { Interruption } 1= & \text { SgNBReleaseTime }+ \text { MeNBReactionTime } \\
& + \text { SgNBAdditionTime }
\end{aligned}
$$

where SgNBReleaseTime represents a time interval between the steps 1 and 8 according to Fig. 5 concerning an overall procedure to release UE context from the SgNB, MeNBReactionTime represents a time interval MeNB needs to check there are some MCG bearers established for the UE that could be reconfigured to SCG ones and initiate SgNB Addition procedure, and SgNBAdditionTime represents a time interval between the steps 1 and 8 according to Fig. 4 concerning an overall procedure to establish UE context at the SgNB. Concerning the SgNBAdditionTime as end point the step 8 was considered because at this point in time data forwarding starts and UE starts to receive data in DL via NR cell.

The interruption time for the alternative 2 was obtained as sum of two sub intervals according the following equation:

Interruption2 = MeNBReactionTime + SgNBModificationTime

where MeNBReactionTime represents a time interval the MeNB, after UE context inactivity info received from SgNB, needs to check there are some MCG bearers established for the UE that could be reconfigured to SCG ones and initiate SgNB modification procedure, and SgNBModificationTime represents a time interval between the steps 1 and 7 according to Fig. 7 concerning an overall procedure to modify UE context at the SgNB. Concerning the SgNBModificationTime as end point the step 7 was considered because at this point in time data forwarding starts and UE starts to receive data in DL via NR cell.

The difference in interruption time between the alternative 1 and 2 is then given as

$$
\begin{gathered}
\text { InterruptionDelta }=(\text { Interruption } 1-\text { Interruption } 2) \\
=(\text { SgNBReleaseTime }+ \text { MeNBReactionTime }+ \text { SgNBAdditionTime }) \\
\quad-(\text { MeNBReactionTime }+ \text { SgNBModificationTime })
\end{gathered}
$$

which results into the following form

$$
\begin{aligned}
\text { InterruptionDelta }= & \text { SgNBReleaseTime }+ \text { SgNBAdditionTime } \\
& - \text { SgNBModificationTime }
\end{aligned}
$$

Note: At the time of writing this paper the procedure related to MeNBReactionTimel has not been yet implemented within the eNB but it is expected to be on the range of tens of ms considering new SgNB detection is

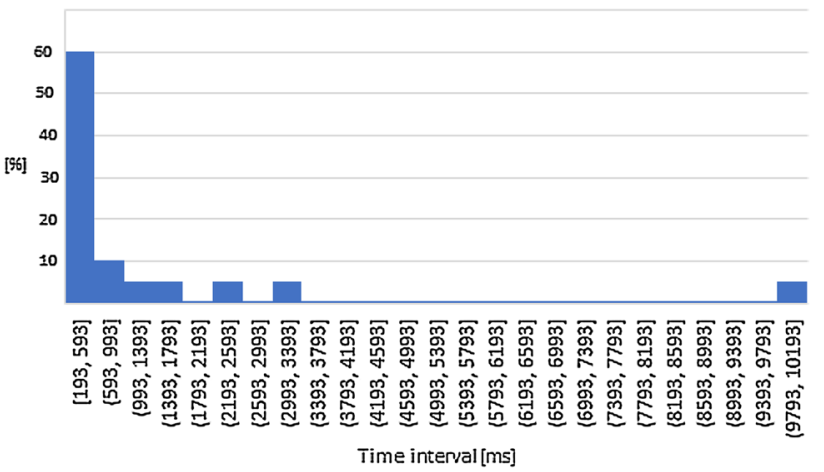

(a)

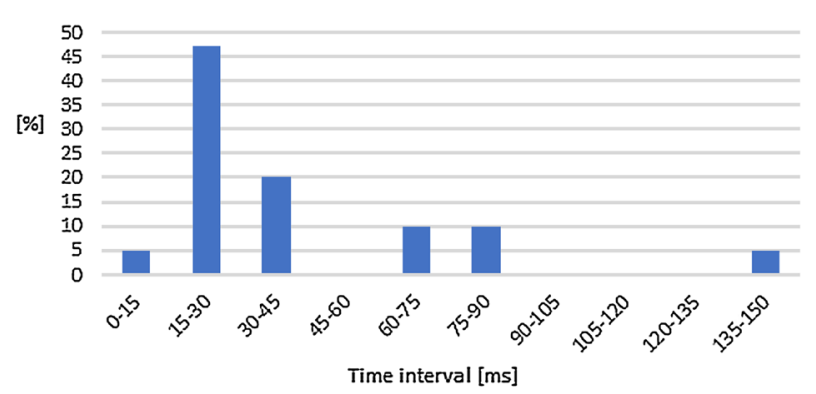

(b)

Fig. 8 Probability distribution function related to: a SgNBAdditionTime and $\mathbf{b}$ SgNBReleaseTime

not needed because SgNB sent SgNB Activity Notification report to MeNB. In case SgNB shall be changed the SgNB follows with SgNB change procedure according to $[10,11]$ and not with SgNB Activity Notification.

The experiments had the following structure. In the first part we run some traces to measure SgNBReleaseTime according to Fig. 5 between the steps 1 and 8 . In the second part we run some traces to measure SgNBAdditionTime according to Fig. 4 between the steps 1 and 8 . In the third part we run some traces to measure SgNBModificationTime between the steps 1 and 8 according to Fig. 7 .

In Fig. 8 a, b probability distribution function related to SgNBAdditionTime and SgNBReleaseTime is shown, respectively. Concerning the SgNBModificationTime the probability distribution function has the similar shape as the one related to SgNBReleaseTime. Corresponding mean values and Eq. 4 applied on these mean values are shown in the Table 2.

Because in case of our experiment the mean values of SgNBModificationTime and SgNBReleaseTime were very similar, the InterruptionDelta calculated according to Eq. 4 was very close to SgNBAdditionTime. In other words, using the alternative 2 the averaged interruption time interval in providing $D C$ services can be reduced by the SgNBAdditionTime. 
Table 2 Average values of SgNBReleaseTime, SgNBAdditionTime, SgNBModificationTime and Eq. 4 obtained from the experiment

\begin{tabular}{llll}
\hline $\begin{array}{l}\text { SgNBRelease- } \\
\text { Time }(\mathrm{ms})\end{array}$ & $\begin{array}{l}\text { SgNBAddition- } \\
\text { Time }(\mathrm{ms})\end{array}$ & $\begin{array}{l}\text { SgNBModifica- } \\
\text { tionTime }(\mathrm{ms})\end{array}$ & Equation 4 (ms) \\
\hline 43 & 1233 & 39 & 1237 \\
\hline
\end{tabular}

\section{Conclusions}

This article gives an overview of the LTE-NR DC feature as standardized in $3 \mathrm{GPP}[6,10,11]$. The architectural options, basic procedures for $D C$ have been discussed in detail. We shed some light on procedure concerning SgNB initiated SgNB release due to user inactivity reason. We propose, the MeNB relies on SgNB Activity Notification reporting according to 3GPP TS 37.340 and follow with reconfiguration of MCG bearers to SCG ones, according to 3GPP TS 36.423, if applicable and being profiting from LTE-NR DC and UE is within the coverage the same SgNB which may completely prevent the SgNB release and thus avoid interruption in providing DC services, guarantee seamless continuation of the LTE-NR DC and reduce number of $\mathrm{X} 2 \mathrm{AP}$ message exchanges between MeNB and SgNB. It is highly recommended to all the vendors focusing on LTENR deployment to follow this approach.

As a future work, further discussions within the 3GPPTS 36.423 to agree on the user story pertaining to this proposal may be expected. In this context, adding a timer in SgNB side after activity notification to give enough time to MeNB for proper reaction as part of 3GPP TS 37.340 standardization may also be anticipated.

Acknowledgements The author acknowledges Mr. Zhongxia Yuan for his help with laboratory testing and doing data analysis.

\section{Compliance with ethical standards}

Conflict of interest The author declares that he has no conflict of interest.

\section{References}

1. Cisco (2016) Cisco visual networking index: global mobile data traffic forecast update, 2015-2020. White paper

2. Antonioli RP, Parente GC, e Silva CF, Rodrigues EB, Maciel TF, Cavalcanti FRP (2017) Dual connectivity for LTE-NR cellular networks. In: Proceeding of the Brazilian telecommunications symposium, pp 1-5
3. Rosa C, Pedersen K, Wang H, Michaelsen PH, Barbera S, Malkamaki E, Henttonen T, Sebire B (2016) Dual connectivity for LTE small cell evolution: functionality and performance aspects. IEEE Commun Mag 54:137-143

4. GPP (2018) Scenarios and requirements of LTE small cell enhancements for E-UTRA and E-UTRAN: 3rd Generation Partnership Project (3GPP) TR 36.932, v.15.0.0

5. Zakrzewska A, López Pérez D, Kucera S, Claussen H (2013) Dual connectivity in LTE HetNets with split control- and user-plane. In: Proceedings of 2013 IEEE Globecom Workshops, pp 391-396

6. GPP (2014) Study on small cell enhancements for E-UTRA and E-UTRAN-higher layer aspects. 3rd Generation Partnership Project (3GPP) TR 36.842, v.12.0.0

7. Kibria MG, Nguyen K, Villardi GP, Ishizu K, Kojima F (2018) Next generation new radio small cell enhancement: architectural options, functionality and performance aspects. IEEE Wirel Commun 25:120-128

8. Yilmaz ONC, Teyeb O, Orsino A (2019) Overview of LTE-NR dual connectivity. IEEE Commun Mag 57:138-144

9. Giordani M, Polese M, Roy A, Castor D, Zorzi M (2019) Standalone and non-standalone beam management for 3GPP NR at mmWaves. IEEE Commun Mag 57:123-129

10. GPP (2018) Multi-connectivity, Stage 2. 3rd Generation Partnership Project (3GPP) TS 37.340, v. 15.3.0

11. GPP (2018) X2 application protocol (X2AP). 3rd Generation Partnership Project (3GPP) TS 36.423, v. 15.3.0

12. Aijaz $A$ (2018) Packet duplication in dual connectivity enabled $5 G$ wireless networks: overview and challenges. arXiv preprint arXiv:1804.01058

13. Samsung (2017) 4G-5G Interworking RAN-level and CN-level Interworking. White paper

14. GPP (2013) Throughput evaluation and comparison of with and without UP bearer split. 3GPP contribution R2-132859

15. GPP (2013) Performance evaluation of user throughput enhancement with multi-stream aggregation over non-ideal backhaul. 3GPP contribution R2-132833

16. Pan MS, Lin TM, Chiu CY, Wang CY (2016) Downlink traffic scheduling for LTE-A small cell networks with dual connectivity enhancement. IEEE Commun Lett 20:796-799

17. ITU (2017) Draft new report ITU-R M. [IMT-2020.TECH PERF $\mathrm{REQ}$ - minimum requirements related to technical performance for IMT-2020 radio interface(s). ITU-R SG05 Contribution 40

18. Capozzi F, Piro G, Grieco LA, Boggia G, Camarda P (2013) Downlink packet scheduling in LTE cellular networks: key design issues and a survey. IEEE Commun Surv Tutor 15:678-700

19. Antonioli R, Parente G, Silva C, Sousa D, Rodrigues E, Maciel T, Cavalcanti F (2018) Dual Connectivity for LTE-NR cellular networks: challenges and open issues. J Commun Inf Syst $33: 282-293$

Publisher's Note Springer Nature remains neutral with regard to jurisdictional claims in published maps and institutional affiliations. 